TRANSACTIONS OF THE

AMERICAN MATHEMATICAL SOCIETY

Volume 348, Number 11, November 1996

\title{
A VARIATIONAL PROBLEM FOR SURFACES IN LAGUERRE GEOMETRY
}

\author{
EMILIO MUSSO AND LORENZO NICOLODI
}

\begin{abstract}
We consider the variational problem defined by the functional $\int \frac{H^{2}-K}{K} d A$ on immersed surfaces in Euclidean space. Using the invariance of the functional under the group of Laguerre transformations, we study the extremal surfaces by the method of moving frames.
\end{abstract}

\section{INTRODUCTION}

In this paper we study the variational problem for the functional

$$
\mathcal{W}(S, f)=\int \frac{H^{2}-K}{K} d A
$$

on immersed surfaces $f: S \rightarrow \mathbb{E}^{3}$ with no parabolic points, where $H$ and $K$ are the mean and Gaussian curvatures of the immersion, and $d A$ is the induced area element. Critical points of this functional were considered by J. Weingarten in 1888 [1] and by W. Blaschke in a series of papers dating from 1924 [2], [3], [4], and in [5]. Since the work of Blaschke, $\mathcal{W}$ has been known to be invariant under the group of Laguerre transformations. These are transformations on the space of oriented spheres which preserve oriented contact of spheres and take planes to planes in $\mathbb{E}^{3}$ (cf. [5], [9]). We intend to study the local geometry of extremal surfaces of $\mathcal{W}$ in the context of Laguerre geometry.

The Laguerre space $\Lambda$ is the space $\mathbb{E}^{3} \times S^{2}$ of contact elements of $\mathbb{E}^{3}$ considered as a homogeneous space of the 10-dimensional Laguerre group $L$ of contact transformations (cf. §1). An immersed surface $f: S \rightarrow \mathbb{E}^{3}$, oriented by a unit normal field $n: S \rightarrow S^{2}$, induces a lift $F=(f, n)$ to $\Lambda$ which is a Legendre immersion with respect to the canonical contact structure of $\Lambda$. We call $F$ the Legendrian lift of $(S, f)$. Locally and up to $L$-congruence, the converse is also true. This intrinsic description of Legendrian lifts arising from immersed surfaces leads to the notion of a Legendre surface, i.e., an immersed surface $F=(f, n): S \rightarrow \Lambda$ such that $d f \cdot n=0$. The additional condition $d n \cdot d n>0$ will be assumed throughout. Moreover, we say that $F$ is nondegenerate if the quadratic forms $d f \cdot d n$ and $d n \cdot d n$ are everywhere linearly independent on $S$.

In Section 1, we develop the method of moving frames in Laguerre geometry to study Legendre surfaces $F: S \rightarrow \Lambda$. We consider the group $L$ as total space of

Received by the editors June 16, 1994.

1991 Mathematics Subject Classification. Primary 58E40, 53A40, 53A05.

Key words and phrases. Laguerre geometry, L-minimal surfaces, Legendre surfaces.

Partially supported by CNR contract n. 93.00554.CTO1, the GADGET initiative of the EC and MURST $40 \%$. 
a principal fiber bundle $\pi_{L}: L \rightarrow \Lambda$ and provide the structure equations for the Laguerre space. If $F$ is nondegenerate, we construct, by successive frame reductions, an essentially unique lifting of $F$ to $L$ and obtain, by pulling back the MaurerCartan form of $L$, the structure equations of the immersion which are given in terms of five invariant functions, $p_{1}, p_{2}, p_{3}, q_{1}, q_{2}$.

On $S$, we define a Laguerre invariant 2 -form $\Omega_{F}$ and consider the variational problem for the functional

$$
\mathcal{W}:(S, F) \rightarrow \int \Omega_{F}
$$

on the space of Legendre immersions. It is shown that

$$
\Omega_{F}=-\frac{H^{2}-K}{K} d A
$$

for any Legendrian lift $F$. Critical points of $\mathcal{W}$, with respect to compactly supported variations, are called $L$-minimal surfaces. On the nondegeneracy locus of a Legendre immersion $F: S \rightarrow \Lambda$, we then construct a Legendre immersion $\check{F}: S \rightarrow \Lambda$, called the Laguerre transform of $F$. In general, $\check{\check{F}} \neq F$.

In Section 2, we compute the Euler-Lagrange equation for the functional $\mathcal{W}$ and express it by the vanishing of the Laguerre invariant $p_{1}+p_{3}$ (Theorem 1 ). In Euclidean terms, this condition is equivalent to the non-linear fourth-order elliptic PDE

$$
\Delta^{\mathrm{III}}\left(\frac{H}{K}\right)=0
$$

where $\Delta^{\mathrm{III}}$ denotes the Laplace-Beltrami operator with respect to the third fundamental form of the surface. In the derivation of the variational equations we proceed in analogy with Bryant [6]. We then consider the uniquely determined complex structure on $S$ compatible with both $\Omega_{F}$ and the conformal class of $d n \cdot d n$. We prove three basic theorems relating the property of being $L$-minimal to this complex structure. In Theorem 2 , from a given nondegenerate $L$-minimal immersion $F: S \rightarrow \Lambda$, we construct a holomorphic quartic differential $\mathcal{P}(F)$ on $S$. In Theorem 3 , we show that a nondegenerate Legendre surface $F: S \rightarrow \Lambda$ is $L$-minimal and $\mathcal{P}(F) \equiv 0$ if and only if the Laguerre transform $\check{F}=(\check{f}, \check{n})$ is totally degenerate, i.e., $\check{f}(S)$ lies in a plane of $\mathbb{E}^{3}$. In Theorem 5 , we then prove that, if $(S, F)$ is $L$-minimal and $\mathcal{P}(F) \not \equiv 0$, the Laguerre transform $\check{F}$ is nondegenerate and $L$-minimal. Moreover, the Laguerre transform of $\check{F}$ gives back $F$. In this case, we say that $\check{F}$ is the Laguerre dual of $F$. We refer to [6] for the conformal analogue.

Section 3 is devoted to the discussion of some special examples of Legendre surfaces. We show that the nondegenerate Legendre surfaces for which the invariant function $p_{2}=0$ are $L$-deformable and that $L$-deformations arise in 1-parameter families (Proposition 6). We prove then in Proposition 7 that $L$-minimal immersions into $\Lambda$ with $p_{2}=0$ and $p_{1}<0$ are locally congruent to the Legendrian lifts of minimal immersions in $\mathbb{E}^{3}$. At last, we obtain Weierstrass-like representation formulae for nondegenerate $L$-minimal surfaces whose holomorphic quartic differential vanishes identically (Proposition 8). 


\section{LAGUERRE GEOMETRY OF LEGENDRE SURFACES}

1.1 The Laguerre space. On $\mathbb{R}^{6}$ with the standard orientation let us consider the scalar product of signature $(4,2)$

$$
\langle X, Y\rangle=-\left(X^{0} Y^{5}+X^{5} Y^{0}\right)-\left(X^{1} Y^{4}+X^{4} Y^{1}\right)+X^{2} Y^{2}+X^{3} Y^{3}=g_{I J} X^{I} Y^{J} .
$$

Let $G$ denote the identity component of the pseudo-orthogonal group of (1.1). The Laguerre group is the subgroup

$$
L=\left\{A=\left(A_{J}^{I}\right) \in G: A_{5}^{J}=0, J=0, \ldots, 4 ; \quad A_{5}^{5}=1\right\} .
$$

It is a 10-dimensional Lie group isomorphic to the identity component of the Poincaré group of the Minkowski 4-space.

Let $\left(e_{0}, \ldots, e_{5}\right)$ be the standard basis of $\mathbb{R}^{6}$. For any $A \in L$, let $A_{J}=A e_{J}$ denote the $J$-th column vector of $A$. $\left(A_{0}, \ldots, A_{5}\right)$ is a Laguerre frame, i.e., a basis of $\mathbb{R}^{6}$ such that

$$
\left\langle A_{I}, A_{J}\right\rangle=g_{I J}, \quad A_{5}=e_{5}, \quad-\left\langle A_{1}+A_{4}, e_{1}+e_{4}\right\rangle \geq 2, \quad I, J=0, \ldots, 5
$$

Regarding the $A_{J}$ 's as $\mathbb{R}^{6}$-valued functions, there exist unique 1-forms $\left\{\omega_{J}^{I}\right\}_{0 \leq I, J \leq 5}$, such that

$$
d A_{I}=\omega_{I}^{J} A_{J}
$$

where $\omega_{J}^{I}$ are the components of the Maurer-Cartan form $\omega=A^{-1} d A$ of $L$. Differentiating (1.2) and (1.3), we get the structure equations of $L$

$$
\begin{gathered}
\omega_{I}^{K} g_{K J}+\omega_{J}^{K} g_{K I}=0, \quad \omega_{5}^{K}=0, \\
d \omega_{J}^{I}=-\omega_{K}^{I} \wedge \omega_{J}^{K} .
\end{gathered}
$$

The Laguerre group acts on the Lie quadric $\mathcal{Q}=\{[X]:\langle X, X\rangle=0\} \subset \mathbb{R} \mathbb{P}^{5}$ by $A \cdot[X]=[A X]$. Besides the "point at infinity" $P_{\infty}=\left[e_{5}\right]$, there are two orbits:

$$
\begin{gathered}
\mathcal{Q}_{\Sigma}=\left\{[X] \in \mathcal{Q}:\left\langle X, e_{5}\right\rangle \neq 0\right\}, \\
\mathcal{Q}_{\Pi}=\left\{[X] \in \mathcal{Q}:\left\langle X, e_{5}\right\rangle=0, X \neq k e_{5}, k \in \mathbb{R}^{*}\right\} .
\end{gathered}
$$

$\mathcal{Q}_{\Sigma}$ is an open dense principal orbit, while $\mathcal{Q}_{\Pi}$ is 3-dimensional. Let $\sigma(p, r)$ denote the oriented sphere in $\mathbb{E}^{3}$ with center $p=\left(p^{1}, p^{2}, p^{3}\right)$ and signed radius $r \in \mathbb{R}$, and $\pi\left(n, p_{0}\right)$ denote the oriented plane through $p_{0}$ orthogonal to $n=\left(n^{1}, n^{2}, n^{3}\right) \in$ $S^{2} \subset \mathbb{E}^{3}$. Then $\mathcal{Q}_{\Sigma}$ is identified with the space of oriented spheres (including point spheres) by $\sigma(p, r) \mapsto\left[{ }^{t}\left(1, \frac{r+p^{1}}{\sqrt{2}}, p^{2}, p^{3}, \frac{r-p^{1}}{\sqrt{2}}, \frac{p \cdot p-r^{2}}{2}\right)\right]$, and $\mathcal{Q}_{\Pi}$ is identified with the space of oriented planes by $\pi\left(n, p_{0}\right) \mapsto\left[{ }^{t}\left(0, \frac{1+n^{1}}{2}, \frac{n^{2}}{\sqrt{2}}, \frac{n^{3}}{\sqrt{2}}, \frac{1-n^{1}}{2}, \frac{n \cdot p_{0}}{\sqrt{2}}\right)\right]$.

Euclidean motions correspond to the elements of $L$ fixing the time-like vector $e_{1}+e_{4}$. Explicitly, for any $(a, p)=\left(\left(a_{J}^{I}\right), p\right) \in \mathbb{E}(3)=S O(3) \rtimes \mathbb{R}^{3}$ we define 
$A(a ; p) \in L$ by

$$
A(a ; p)=\left(\begin{array}{cccccc}
1 & 0 & 0 & 0 & 0 & 0 \\
\frac{p^{1}}{\sqrt{2}} & \frac{1+a_{1}^{1}}{2} & \frac{a_{2}^{1}}{\sqrt{2}} & \frac{a_{3}^{1}}{\sqrt{2}} & \frac{1-a_{1}^{1}}{2} & 0 \\
p^{2} & \frac{a_{1}^{2}}{\sqrt{2}} & a_{2}^{2} & a_{3}^{2} & \frac{-a_{1}^{2}}{\sqrt{2}} & 0 \\
p^{3} & \frac{a_{1}^{3}}{\sqrt{2}} & a_{2}^{3} & a_{3}^{3} & \frac{-a_{1}^{3}}{\sqrt{2}} & 0 \\
\frac{-p^{1}}{\sqrt{2}} & \frac{1-a_{1}^{1}}{2} & \frac{-a_{2}^{1}}{\sqrt{2}} & \frac{-a_{3}^{1}}{\sqrt{2}} & \frac{1+a_{1}^{1}}{2} & 0 \\
\frac{p \cdot p}{2} & \frac{a_{1}^{J} p^{J}}{\sqrt{2}} & a_{2}^{J} p^{J} & a_{3}^{J} p^{J} & \frac{-a_{1}^{J} p^{J}}{\sqrt{2}} & 1
\end{array}\right) .
$$

The projective line $[X, Y]$ determined by the points $[X],[Y] \in \mathcal{Q}$ lies on $\mathcal{Q}$ if and only if $\langle X, Y\rangle=0$. This means that the geometric objects in $\mathbb{E}^{3}$ corresponding to $[X]$ and $[Y]$ are in oriented contact. Note that for $A=\left(A_{0}, \ldots, A_{5}\right) \in L$, the oriented planes represented by $\left[A_{1}\right],\left[A_{4}\right]$ are in oriented contact with the oriented sphere represented by $\left[A_{0}\right]$.

The Laguerre space $\Lambda$ is the space of lines $\ell \subset \mathcal{Q}$ which do not meet the point at infinity $P_{\infty}$. $L$ acts transitively on $\Lambda$ and the map $\pi_{L}: L \rightarrow \Lambda, A \mapsto\left[A_{0}, A_{1}\right]$, makes $L$ into a principal fiber bundle over $\Lambda$ (the Laguerre fibration) with fiber $L_{0}$ whose elements are matrices of the form

$$
X(d ; b ; x)=\left(\begin{array}{cccccc}
1 & 0 & 0 & 0 & 0 & 0 \\
d_{1} & d_{2} & \tilde{x}^{1} & \tilde{x}^{2} & {\frac{d_{2}}{2}}^{t} x x & 0 \\
0 & 0 & b_{1}^{1} & b_{2}^{1} & x^{1} & 0 \\
0 & 0 & b_{1}^{2} & b_{2}^{2} & x^{2} & 0 \\
0 & 0 & 0 & 0 & \frac{1}{d_{2}} & 0 \\
0 & 0 & 0 & 0 & -\frac{d_{1}}{d_{2}} & 1
\end{array}\right),
$$

where $b=\left(b_{j}^{i}\right) \in S O(2), d=\left(d_{1}, d_{2}\right) \in \mathbb{R}^{2}, d_{2} \neq 0, x={ }^{t}\left(x^{1}, x^{2}\right) \in \mathbb{R}^{2},\left(\tilde{x}^{1}, \tilde{x}^{2}\right)=$ $d_{2}{ }^{t} x b$ and $\frac{1}{d_{2}}\left(1+d_{2}{ }^{2}\right)+\frac{1}{2} d_{2}{ }^{t} x x \geq 2$.

Every line $\ell \in \Lambda$ contains a unique point $p(\ell) \in \mathbb{E}^{3}$ and a unique oriented plane $\pi$ through $p(\ell)$. Let $n(\ell)$ denote the unit normal vector of $\pi$. $\Lambda$ is identified with the unit tangent bundle $\mathbb{E}^{3} \times S^{2}$ by the correspondence

$$
\Lambda \ni \ell \mapsto(p(\ell), n(\ell)) \in \mathbb{E}^{3} \times S^{2},
$$

and $L$ can be seen as a 10-dimensional group of contact transformations acting on $\mathbb{E}^{3} \times S^{2}$ (cf. [5], [9] for more details about Laguerre geometry).

1.2 Adapted frames. Let $F=(f, n): S \rightarrow \Lambda$ be a Legendre immersion. A local Laguerre frame field along $(S, F)$ is a smooth map $A: \mathcal{U} \subset S \rightarrow L$ defined on an open subset $\mathcal{U}$ of $S$ such that $\pi_{L}(A(s))=F(s)$, for each $s \in \mathcal{U}$. For any Laguerre frame field $A: \mathcal{U} \rightarrow L$ we let $\alpha_{J}^{I}=A^{*}\left(\omega_{J}^{I}\right), I, J=0,1, \ldots, 5$. We then have

$$
\alpha_{0}^{4}=0, \quad \alpha_{1}^{2} \wedge \alpha_{1}^{3} \neq 0 .
$$

Differentiating $\alpha_{0}^{4}=0$ and using (1.5) we get $\alpha_{0}^{2} \wedge \alpha_{1}^{2}+\alpha_{0}^{3} \wedge \alpha_{1}^{3}=0$. By Cartan's Lemma, there exist smooth functions $M_{i j}=M_{j i}, 1 \leq i, j \leq 2$, such that

$$
\alpha_{0}^{2}=M_{11} \alpha_{1}^{2}+M_{12} \alpha_{1}^{3}, \quad \alpha_{0}^{3}=M_{12} \alpha_{1}^{2}+M_{22} \alpha_{1}^{3} .
$$

Any other Laguerre frame field $\hat{A}$ on $\mathcal{U}$ is given by $\hat{A}=A X(d ; b ; x)$, where $X=$ $X(d ; b ; x): \mathcal{U} \rightarrow L_{0}$ is a smooth map. Thus $\hat{\alpha}$ and $\alpha$ are related by $\hat{\alpha}=X^{-1} \alpha X+$ 
$X^{-1} d X$. This implies

$$
\left(\begin{array}{c}
\hat{\alpha}_{0}^{2} \\
\hat{\alpha}_{0}^{3}
\end{array}\right)={ }^{t} b\left(\begin{array}{c}
\alpha_{0}^{2} \\
\alpha_{0}^{3}
\end{array}\right)+d_{1}{ }^{t} b\left(\begin{array}{c}
\alpha_{1}^{2} \\
\alpha_{1}^{3}
\end{array}\right), \quad\left(\begin{array}{c}
\hat{\alpha}_{1}^{2} \\
\hat{\alpha}_{1}^{3}
\end{array}\right)=d_{2}{ }^{t} b\left(\begin{array}{c}
\alpha_{1}^{2} \\
\alpha_{1}^{3}
\end{array}\right) .
$$

Using (1.7) and (1.8) we get

$$
\left(\begin{array}{ll}
\hat{M}_{11} & \hat{M}_{12} \\
\hat{M}_{12} & \hat{M}_{22}
\end{array}\right)=\frac{1}{d}^{t} b\left(\begin{array}{cc}
M_{11}+d_{1} & M_{12} \\
M_{12} & M_{22}+d_{1}
\end{array}\right) b .
$$

Therefore,

$$
\frac{1}{2}\left(\hat{M}_{11}+\hat{M}_{22}\right)=\frac{1}{d_{2}}\left[\frac{1}{2}\left(M_{11}+M_{22}\right)+d_{1}\right] .
$$

This shows that, locally, there exist Laguerre frames such that

$$
M_{11}+M_{22}=0
$$

A Laguerre frame field satisfying (1.11) is said to be of first order. By (1.10), it follows that if $A$ is a first order frame field, then any other first order frame on $\mathcal{U}$ is given by $A X(d ; b ; x)$, where $X: \mathcal{U} \rightarrow L_{1}$ is a smooth map, and

$$
L_{1}=\left\{X(d ; b ; x) \in L_{0}: d_{1}=0\right\} .
$$

Remark. A sphere congruence is a 2-parameter family of oriented spheres, i.e., a smooth map $\sigma: S \rightarrow \mathcal{Q}_{\Sigma}$ of a connected surface into the space of oriented spheres. By an envelope of $\sigma: S \rightarrow \mathcal{Q}_{\Sigma}$ we mean a Legendre map $F=(f, n): S \rightarrow \Lambda$ such that $\sigma(s)$ and the plane $\pi(n(s), f(s))$ are in oriented contact at $f(s)$, for every $s \in S$.

Note that the map $\sigma_{F}: \mathcal{U} \rightarrow \mathcal{Q}_{\Sigma}, s \mapsto\left[A_{0}(s)\right]$ is independent of the first order frame $A$. This define a sphere congruence which is Laguerre-invariantly connected with $F$, called the central congruence (Laguerre spherical map) of $(S, F)$. By construction, the Legendre immersion $(S, F)$ is an envelope of the central congruence.

Using first order frames, (1.8) and (1.9), we see that the immersion $F$ induces on $S$ a quadratic form $\Phi_{F}=\left(\alpha_{0}^{2}\right)^{2}+\left(\alpha_{0}^{3}\right)^{2}$, the Laguerre line element, and an exterior differential 2-form $\Omega_{F}=\alpha_{0}^{2} \wedge \alpha_{0}^{3}$, the Laguerre area element of the immersion. The nondegeneracy of $F$ means that $\Phi_{F}$ is positive definite and $\Omega_{F \mid s} \neq 0$, for each $s \in S$.

Under the nondegeneracy assumption, for any first order frame $A: \mathcal{U} \rightarrow L$ we set

$$
\alpha_{0}^{1}=M_{0}^{1} \alpha_{0}^{2}+N_{0}^{1} \alpha_{0}^{3},
$$

where $M_{0}^{1}$ and $N_{0}^{1}$ are smooth functions depending on $A$. If $\hat{A}=A X(d ; b ; x)$ is any other first order frame on $\mathcal{U}$, then

$$
\hat{\alpha}_{0}^{1}=\frac{1}{d_{2}}\left(\alpha_{0}^{1}-d_{2} x^{1} \alpha_{0}^{2}-d_{2} x^{2} \alpha_{0}^{3}\right),
$$

and hence, by (1.8),

$$
\left(\begin{array}{c}
\hat{M}_{0}^{1} \\
\hat{N}_{0}^{1}
\end{array}\right)=\frac{1}{d_{2}} t b\left(\begin{array}{c}
M_{0}^{1}-d_{2} x^{1} \\
N_{0}^{1}-d_{2} x^{2}
\end{array}\right)
$$

It follows that, for any point $s \in S$, there exists an open neighborhood of $s$ on which there is defined a first order frame $A$ such that $M_{0}^{1}=N_{0}^{1}=0$, i.e., $\alpha_{0}^{1}=0$. 
A first order Laguerre frame field is of second order if $\alpha_{0}^{1}=0$. If $A: \mathcal{U} \rightarrow L$ is a second order frame field, then any other second order frame on $\mathcal{U}$ is given by $\hat{A}=A X$, where $X: \mathcal{U} \rightarrow L_{2} \subset L_{1}$ is a smooth map, and

$$
L_{2}=\left\{X(d ; b ; x): d_{1}=x^{1}=x^{2}=0\right\} .
$$

Remark. From this we infer that the projective line $\left[A_{0}(s), A_{4}(s)\right]$ does not depend on the choice of second order frames. Thus we may define a new Legendre immersion $\check{F}: S \rightarrow \Lambda$, associated with $F$, by

$$
\check{F}(s)=\left[A_{0}(s), A_{4}(s)\right] \text {, for each } s \in S .
$$

We call $\check{F}$ the Laguerre transform of $F$. We remark that $\check{F}$ is the second envelope of the central congruence.

From (1.9) it follows that there exist second order frames $A$ defined near any point of $S$ such that

$$
M_{11}=-M_{22}=1, \quad M_{12}=0 .
$$

A second order frame field with this property is called a normal frame along $(S, F)$. Two normal frames $A, \hat{A}: \mathcal{U} \rightarrow L$ are related by $\hat{A}=A X$, where $X \in \mathbb{Z}_{2}$, and

$$
\mathbb{Z}_{2}=\left\{X(d ; b ; x) \in L_{0}: d_{1}=x^{1}=x^{2}=0, d_{2}=1, b= \pm\left(\begin{array}{ll}
1 & 0 \\
0 & 1
\end{array}\right)\right\} .
$$

The totality of normal frames on $S$ is the $\mathbb{Z}_{2}$-principal bundle $\pi_{F}: \mathcal{F}(F) \rightarrow S$, where

$$
\mathcal{F}(F)=\{(s, A(s)) \in S \times L\}
$$

and $A$ is any normal frame field along $F$ defined in a neighborhood of $s$, and $\pi_{F}:(s, A(s)) \in \mathcal{F}(F) \mapsto s \in S$.

Remark. Two Legendrian surfaces $(S, F)$ and $\left(S^{\prime}, F^{\prime}\right)$ are said to be $L$-equivalent if there exists $A \in L$ such that $A F^{\prime}\left(S^{\prime}\right)=F(S)$. We observe that, up to $L$ equivalence, any nondegenerate Legendrian surface admits a globally defined normal frame. In fact, consider a connected component $\tilde{S}$ of $\mathcal{F}(F)$ and let $\tilde{F}: \tilde{S} \rightarrow \Lambda$ be given by $\tilde{F}=F \circ \pi_{F}$. By construction, $\tilde{F}$ is again a nondegenerate Legendre immersion and $F(S)=\tilde{F}(\tilde{S})$. Moreover, $(s, A(s)) \in \tilde{S} \rightarrow A(s) \in L$ is a global normal frame along $(\tilde{S}, \tilde{F})$.

Let $A: S \rightarrow L$ be a normal frame along $(S, F)$. The above discussion allows us to set

$$
\begin{gathered}
\alpha_{2}^{1}=p_{1} \alpha_{0}^{2}+p_{2} \alpha_{0}^{3}, \quad \alpha_{3}^{1}=p_{2} \alpha_{0}^{2}+p_{3} \alpha_{0}^{3}, \\
\alpha_{2}^{3}=q_{1} \alpha_{0}^{2}+q_{2} \alpha_{0}^{3} .
\end{gathered}
$$

where $p_{1}, p_{2}, p_{3}$ and $q_{1}, q_{2}$ are real-valued smooth functions. We call $p_{1}, p_{2}, p_{3}, q_{1}, q_{2}$ the invariant functions and $\left(\alpha_{0}^{2}, \alpha_{0}^{3}\right)$ the normal coframing of the Legendre immersion (with respect to the given normal frame field $A$ ).

From the structure equations (1.5) we compute $\alpha_{1}^{1}=2 q_{2} \alpha_{0}^{2}-2 q_{1} \alpha_{0}^{3}$ and

$$
d \alpha_{0}^{2}=q_{1} \alpha_{0}^{2} \wedge \alpha_{0}^{3}, \quad d \alpha_{0}^{3}=q_{2} \alpha_{0}^{2} \wedge \alpha_{0}^{3},
$$




$$
\begin{aligned}
& d q_{1} \wedge \alpha_{0}^{2}+d q_{2} \wedge \alpha_{0}^{3}=\left(p_{3}-p_{1}-q_{1}^{2}-q_{2}^{2}\right) \alpha_{0}^{2} \wedge \alpha_{0}^{3}, \\
& d q_{1} \wedge \alpha_{0}^{3}-d q_{2} \wedge \alpha_{0}^{2}=-p_{2} \alpha_{0}^{2} \wedge \alpha_{0}^{3}, \\
& d p_{1} \wedge \alpha_{0}^{2}+d p_{2} \wedge \alpha_{0}^{3}=\left(-3 q_{1} p_{1}-4 q_{2} p_{2}+q_{1} p_{3}\right) \alpha_{0}^{2} \wedge \alpha_{0}^{3}, \\
& d p_{2} \wedge \alpha_{0}^{2}+d p_{3} \wedge \alpha_{0}^{3}=\left(-3 q_{2} p_{3}-4 q_{1} p_{2}+q_{2} p_{1}\right) \alpha_{0}^{2} \wedge \alpha_{0}^{3} .
\end{aligned}
$$

(1.14) and (1.15) will be referred to as the structure equations of the Legendre immersion.

1.3 Relations with Euclidean geometry. Let $F=(f, n): S \rightarrow \Lambda$ be the Legendrian lift of an oriented surface $f: S \rightarrow \mathbb{E}^{3}$ and suppose that $f$ is umbilic-free and that its Gaussian curvature is everywhere different from zero. We may assume the existence of a global principal framing $e=\left(n, e_{2}, e_{3} ; f\right): S \rightarrow \mathbb{E}(3)$ along $f$, where $\left(e_{2}, e_{3}\right)_{s}$ is a positive basis of $d f_{s}\left(T_{s} S\right)$, for each $s \in S$, and $e_{2}, e_{3}$ are along the principal directions corresponding to the principal curvatures $\kappa_{2}, \kappa_{3}$ with $\kappa_{2}>\kappa_{3}$. We have then

$$
d f=\theta^{2} e_{2}+\theta^{3} e_{3}, \quad d n=\theta_{1}^{2} e_{2}+\theta_{1}^{3} e_{3}, \quad d e_{2}=\theta_{2}^{3} e_{3}-\theta_{1}^{2} n, \quad d e_{3}=-\theta_{2}^{3} e_{2}-\theta_{1}^{3} n,
$$

where $\left(\theta^{2}, \theta^{3}\right)$ is the coframe on $S$ and $\theta_{1}^{2}=-\kappa_{2} \theta^{2}, \quad \theta_{1}^{3}=-\kappa_{3} \theta^{3}$.

According to (1.6), the principal framing $e$ gives rise to a Laguerre frame field $A\left(n, e_{2}, e_{3} ; f\right)$ along the Legendrian lift of $f$. We compute

$$
\begin{aligned}
& d A_{0}=\theta^{2} A_{2}+\theta^{3} A_{3}, \quad d A_{1}=\frac{\theta_{1}^{2}}{\sqrt{2}} A_{2}+\frac{\theta_{1}^{3}}{\sqrt{2}} A_{3}, \\
& d A_{2}=-\frac{\theta_{1}^{2}}{\sqrt{2}} A_{1}+\theta_{2}^{3} A_{3}+\frac{\theta_{1}^{2}}{\sqrt{2}} A_{4}+\theta^{2} A_{5}, \\
& d A_{3}=-\frac{\theta_{1}^{3}}{\sqrt{2}} A_{1}-\theta_{2}^{3} A_{2}+\frac{\theta_{1}^{3}}{\sqrt{2}} A_{4}+\theta^{3} A_{5}, \\
& d A_{4}=-\frac{\theta_{1}^{2}}{\sqrt{2}} A_{2}-\frac{\theta_{1}^{3}}{\sqrt{2}} A_{3}, \quad d A_{5}=0 .
\end{aligned}
$$

Thus, $\alpha_{0}^{2}=-\frac{\sqrt{2}}{\kappa_{2}} \alpha_{1}^{2}, \alpha_{0}^{3}=-\frac{\sqrt{2}}{\kappa_{3}} \alpha_{1}^{3}$, and following the reduction procedure described above, we may adapt $A$ to a first order Laguerre frame $A^{\prime}$ along $(S, F)$ by setting

$$
A^{\prime}=A X\left(d ; I_{2 \times 2} ; 0\right), \text { with } d=\left(\sqrt{2} \frac{H}{K}, 1\right),
$$

where $H=\frac{1}{2}\left(\kappa_{2}+\kappa_{3}\right)$ and $K=\kappa_{2} \kappa_{3}$ denote, respectively, the mean curvature and the Gaussian curvature of $f$. So

$$
\alpha_{0}^{\prime 2}=\frac{\kappa_{2}-\kappa_{3}}{2 K} \theta_{1}^{2}, \quad \alpha_{0}^{\prime 3}=\frac{\kappa_{3}-\kappa_{2}}{2 K} \theta_{1}^{3} .
$$

Accordingly, the Laguerre line element and the Laguerre area element take the form

$$
\Phi_{F}=\frac{H^{2}-K}{K^{2}} d n \cdot d n, \quad \Omega_{F}=-\frac{H^{2}-K}{K} d A,
$$

respectively, where $d A$ is the Euclidean area element of $f$. Therefore, for the Legendrian lift of an oriented surface $f: S \rightarrow \mathbb{E}^{3}$, the nondegeneracy condition means that $f$ is umbilic free and its Gaussian curvature is everywhere different from zero. It is worth noting that the Euclidean invariant $\frac{H^{2}-K}{K} d A$ is actually a Laguerre invariant. 
It is also easily seen that the central sphere congruence is given by the oriented spheres $\sigma\left(f(s)+\frac{H}{K} n(s), \frac{H}{K}\right)$, with center $f(s)+\frac{H}{K} n(s)$ and signed radius $\frac{H}{K}(s)$.

\section{L-MINIMAL SURFACES}

2.1 The Weingarten functional. Let $F: S \rightarrow \Lambda$ be a Legendre surface. A compactly supported variation of $(S, F)$ is a differentiable mapping

$$
V: S \times(-\epsilon, \epsilon) \rightarrow \Lambda, \quad(s, t) \mapsto V(s, t), \quad \epsilon>0,
$$

such that its restriction $F_{t}$ to $S \times t, t \in(-\epsilon, \epsilon)$, is a Legendre surface, $F_{0}=F$, and such that there exists a compact domain $K \subset S$ for which $V(s, t)=F(s)$, for all $s \in S-K$ and all $t \in(-\epsilon, \epsilon)$. If $F$ is nondegenerate we may suppose that $F_{t}$ is also nondegenerate, for each $t \in(-\epsilon, \epsilon)$.

Given a compact domain $K \subset S$, we define the functional

$$
\mathcal{W}_{K}(F)=\int_{K} \Omega_{F}
$$

on the space of smooth Legendre immersions $F: S \rightarrow \Lambda$. We call (2.1) the Weingarten functional. A Legendre surface $F: S \rightarrow \Lambda$ is an L-minimal surface if it is an extremal of (2.1), that is to say, if for any compact domain $K \subset S$ and any differentiable variation $F_{t}$ with support in $K$ we have

$$
\frac{d}{d t}\left(\mathcal{W}_{K}\left(F_{t}\right)\right)_{\mid t=0}=0
$$

The purpose of this section is to calculate the Euler-Lagrange equation for this variational problem in an $L$-invariant way. We do this by the method of moving frame [8], [10].

Let $V$ be a smooth variation of $F: S \rightarrow \Lambda$ with support in a compact set $K \subset S$. Assuming that $F_{t}$ is nondegenerate for each $t \in(-\epsilon, \epsilon)$, we may construct (cf. preceding section) a $\mathbb{Z}_{2}$-bundle over $S \times(-\epsilon, \epsilon)$

$$
\pi_{V}: \mathcal{F}(V) \rightarrow S \times(-\epsilon, \epsilon),
$$

where $\mathcal{F}(V) \subset S \times(-\epsilon, \epsilon) \times L$ is defined by

$$
\mathcal{F}(V)=\left\{(s, t, A):(s, A) \in \pi_{F_{t}}^{-1}(s)\right\},
$$

and $\pi_{F_{t}}(s): \mathcal{F}\left(F_{t}\right) \rightarrow S$ is the fibering of normal Laguerre frame fields along $F_{t}$. A local cross section of $(2.2)$ is a smooth map $A: \mathcal{U} \times I \rightarrow L$, defined on some open subset $\mathcal{U} \times I$ of $S \times(-\epsilon, \epsilon)$, such that $A_{t}: s \in \mathcal{U} \mapsto A(s, t) \in L$ is a normal frame field along $F_{t}$. Local sections of (2.2) are called Laguerre frame fields of the variation.

For any such frame field $A: \mathcal{U} \times I \rightarrow L$ we set $\alpha=A^{*}(\omega)=\left(\alpha_{J}^{I}\right)$. The entries of the matrix-valued 1-form $\alpha$ are then determined by

$$
\alpha_{I}^{K} g_{K J}+\alpha_{J}^{K} g_{K I}=0, \quad \alpha_{5}^{K}=0
$$

and

$$
\begin{aligned}
& \alpha_{0}^{1}=a_{0}^{1} d t, \quad \alpha_{1}^{1}=2 q_{2} \alpha_{0}^{2}-2 q_{1} \alpha_{0}^{3}+a_{1}^{1} d t, \quad \alpha_{2}^{1}=p_{1} \alpha_{0}^{2}+p_{2} \alpha_{0}^{3}+a_{2}^{1} d t \\
& \alpha_{3}^{1}=p_{2} \alpha_{0}^{2}+p_{3} \alpha_{0}^{3}+a_{3}^{1} d t, \quad \alpha_{1}^{2}=\alpha_{0}^{2}+a_{1}^{2} d t, \quad \alpha_{1}^{3}=-\alpha_{0}^{3}+a_{1}^{3} d t \\
& \alpha_{2}^{3}=q_{1} \alpha_{0}^{2}+q_{2} \alpha_{0}^{3}+a_{2}^{3} d t, \quad \alpha_{0}^{4}=a_{0}^{4} d t
\end{aligned}
$$

where

$$
\alpha_{0}^{2} \wedge \alpha_{0}^{3} \wedge d t_{\left.\right|_{(s, t)}} \neq 0, \quad \text { for all }(s, t) \in \mathcal{U} \times I
$$


The functions $q_{i}(\cdot, t), p_{j}(\cdot, t)$ are the invariant functions of $F_{t}$, and $a_{J}^{I}(s, t)=0$ for all $s \in \mathcal{U}-\mathcal{U} \cap K, t \in I$.

We now consider the exterior differential 2-form

$$
\delta \Omega_{V}=\left(p_{1}+p_{3}\right) \alpha_{0}^{2} \wedge \alpha_{0}^{3}
$$

and we set

$$
\lambda_{V}=a_{0}^{4} .
$$

It is easily seen that $\delta \Omega_{V}$ and $\lambda_{V}$ do not depend on the choice of the Laguerre frame. Thus $\delta \Omega_{V}$ and $\lambda_{V}$ are defined on $S \times(-\epsilon, \epsilon)$ by the variation $V$.

If $\mathcal{U}$ is an open connected subset of $S-K$ and $I$ an interval in $(-\epsilon, \epsilon)$, any Laguerre frame of the variation $A: \mathcal{U} \times I \rightarrow L$ does not depend on the parameter $t$, i.e., $\frac{\partial A}{\partial t}=0$. Therefore, if we define $\psi_{V}$ by

$$
\psi_{V}=\imath_{\frac{\partial}{\partial t}}\left(\alpha_{0}^{2} \wedge \alpha_{0}^{3}\right)
$$

then $\psi_{\left.V\right|_{(s, t)}}=0$, for all $(s, t) \in S-K \times(-\epsilon, \epsilon)$. In particular $\psi_{\left.V\right|_{S \times\{0\}}}$ is a 1-form with support in $K$, and $\lambda_{\left.V\right|_{S \times\{0\}}}$ is a function with the same property.

We now make the following remark:

(2.7) Let $F: S \rightarrow \Lambda$ be a nondegenerate Legendre immersion. For any point $s_{0} \in S$ there exists an open neighborhood $\mathcal{U}$ of $s_{0}$ such that, for any smooth function $\lambda: S \rightarrow \mathbb{R}$ with support contained in a compact set $D \subset \mathcal{U}$, there is a variation $V$ of $F$ with support in $D$ verifying $\lambda_{\left.V\right|_{S \times\{0\}}}=\lambda$.

This technical property can be justified as follows: given a point $s_{0}$ there exists an element $A \in L$ such that the Euclidean projection of $A F$ is a smooth immersion on an open neighborhood of $s_{0}$.

Thus we may suppose from the beginning that the Euclidean projection of $F$ is a smooth immersion on a simply connected open neighborhood $\mathcal{U}$ of $s_{0}$. Moreover, if we let $K$ and $\kappa_{2}>\kappa_{3}$ be the Gaussian curvature and the principal curvatures, respectively, then $K(s) \neq 0$ and $\kappa_{2}(s) \neq \kappa_{3}(s)$, for all $s \in \mathcal{U}$. Given the function $\lambda$ we consider $\tilde{\lambda}=\frac{\sqrt{2} K}{\kappa_{2}-\kappa_{3}} \lambda$. This is again a function with support contained in $D$. Then there exists a variation $G_{t}: \mathcal{U} \times(-\epsilon, \epsilon) \rightarrow \mathbb{E}^{3}$ of $f$ so that $\tilde{\lambda} n$ is the variation vector field at $t=0$.

Let $N_{t}: \mathcal{U} \times(-\epsilon, \epsilon) \rightarrow S^{2}$ be defined by the unit normal of $G_{t}: \mathcal{U} \rightarrow \mathbb{E}^{3}$ at $G_{t}(s)$. Then, the mapping

$$
V=\left(G_{t}, N_{t}\right): \mathcal{U} \times(-\epsilon, \epsilon) \rightarrow \Lambda
$$

is a variation of $F_{l_{\mathcal{U}}}$ with the required properties. Extending $V$ to the whole of $S \times(-\epsilon, \epsilon)$ by setting $V(s, t)=F(s)$ for all $s \in S-\mathcal{U}$ and $t \in(-\epsilon, \epsilon)$, we obtain the required result.

We are now ready to prove

Theorem 1. A nondegenerate Legendre surface $F: S \rightarrow \Lambda$ is L-minimal if and only if $p_{1}+p_{3}=0$.

Proof. Let $V: S \times(-\epsilon, \epsilon) \rightarrow \Lambda$ be a smooth variation of $F$ with support in the compact set $K \subset S$. We claim that

$$
\frac{d}{d t}\left(\mathcal{W}_{K}\left(F_{t}\right)\right)_{\left.\right|_{t=0}}=\int_{K} \lambda_{\left.V\right|_{S \times\{0\}}} \delta \Omega_{\left.V\right|_{S \times\{0\}}} .
$$


For each $t \in(-\epsilon, \epsilon)$ we let $j_{t}: s \in S \mapsto(s, t) \in S \times(-\epsilon, \epsilon)$ and we remark that

$$
\Omega_{F_{t}}=j_{t}^{*}\left(\Psi_{V}\right), \quad \text { where } \Psi_{V}=\alpha_{0}^{2} \wedge \alpha_{0}^{3} .
$$

Thus we have

$$
\frac{d}{d t}\left(\mathcal{W}_{K}\left(F_{t}\right)\right)_{\mid t=0}=\frac{d}{d t}\left[\int_{K} j_{t}^{*}\left(\Psi_{V}\right)\right]_{\mid t=0}=\int_{K} j_{0}^{*}\left(L_{\frac{\partial}{\partial t}} \Psi_{V}\right) .
$$

Cartan's formula yields

$$
j_{0}^{*}\left(L_{\frac{\partial}{\partial t}} \Psi_{V}\right)=j_{0}^{*}\left(\iota_{\frac{\partial}{\partial t}} d \Psi_{V}+d \psi_{V}\right)=j_{0}^{*}\left(\iota_{\frac{\partial}{\partial t}} d \Psi_{V}\right)+d j_{0}^{*}\left(\psi_{V}\right)
$$

Note that $d j_{0}^{*}\left(\psi_{V}\right)$ is an exact form and $j_{0}^{*}\left(\psi_{V}\right)$ has support in the compact $K$. By substituting (2.10) into (2.9), we obtain using Stokes' theorem

$$
\frac{d}{d t}\left(\mathcal{W}_{K}\left(F_{t}\right)\right)_{t=0}=\int_{K} j_{0}^{*}\left(\imath_{\partial t} d \Psi_{V}\right) .
$$

From the structure equation $d \alpha=-\alpha \wedge \alpha$ we compute

$$
d \Psi_{V}=a_{0}^{4}\left(p_{1}+p_{3}\right) d t \wedge \Psi_{V} .
$$

Accordingly,

$$
\frac{d}{d t}\left(\mathcal{W}_{K}\left(F_{t}\right)\right)_{\left.\right|_{t=0}}=\int_{K} j_{0}^{*}\left(\lambda_{V}\right)\left(p_{1}+p_{3}\right) \Omega_{F}
$$

Thus, by (2.7), the left-hand side of (2.11) vanishes if and only if $\delta \Omega_{V}=0$.

Remark. a) To interpret the condition $p_{1}+p_{3}=0$ in terms of classical Euclidean invariants we carry out the relationship with Euclidean surface theory begun in $\S 1.3$. In our calculation, it is convenient to make use of the coframe $\left(\theta_{1}^{2}, \theta_{1}^{3}\right)$. We let

and compute

$$
d\left(\frac{H}{K}\right)=\frac{\kappa_{3}-\kappa_{2}}{2 K}\left(\left(\frac{H}{K}\right)_{2} \theta_{1}^{2}+\left(\frac{H}{K}\right)_{3} \theta_{1}^{3}\right)
$$

$$
\alpha^{\prime 1}=-\sqrt{2}\left(\frac{H}{K}\right)_{2} \alpha_{0}^{\prime 2}+\sqrt{2}\left(\frac{H}{K}\right)_{3} \alpha_{0}^{\prime 3} .
$$

We then adapt $A^{\prime}$ further to a second order frame

$$
A^{\prime \prime}=A^{\prime} X\left(d ; I_{2 \times 2} ; x\right), \quad d=(0,1), \quad x={ }^{t}\left(-\sqrt{2}\left(\frac{H}{K}\right)_{2}, \sqrt{2}\left(\frac{H}{K}\right)_{3}\right) .
$$

$A^{\prime \prime}$ originates a normal frame and the corresponding normal coframe is $-\frac{\kappa_{3}-\kappa_{2}}{2 K} \theta_{1}^{2}$, $\frac{\kappa_{3}-\kappa_{2}}{2 K} \theta_{1}^{3}$. Using (1.12) we write $p_{1}, p_{3}$ and, by means of the integrability conditions

$$
d \theta_{1}^{2}=\theta_{2}^{3} \wedge \theta_{1}^{3}, \quad d \theta_{1}^{3}=\theta_{1}^{2} \wedge \theta_{2}^{3},
$$

one can show that the condition to be $L$-minimal is expressed by

$$
\Delta^{\mathrm{III}}\left(\frac{H}{K}\right)=0
$$

where $\Delta^{\mathrm{III}}$ denotes the Laplace-Beltrami operator with respect to the third fundamental form III $=\left(\theta_{1}^{2}\right)^{2}+\left(\theta_{1}^{3}\right)^{2}$ of the surface. In this form, the Euler-Lagrange equation for the functional $\mathcal{W}(S, f)=\int \frac{H^{2}-K}{K} d A$ has been known since Blaschke [4], [5]. One can show that the exterior differential system whose solutions are the $L$-minimal surfaces is involutive in Cartan's sense (cf. [7]).

b) $\mathcal{Q}_{\Sigma}$ is diffeomorphic to the Minkowski 4 -space $\mathbb{R}_{1}^{4}$ spanned by $e_{1}, e_{2}, e_{3}, e_{4}$ and with scalar product obtained by restriction of $\langle$,$\rangle . Thus, the central congruence of$ 
a Legendre immersion $(F, S)$ may be regarded as a smooth mapping $\sigma_{F}: S \rightarrow \mathbb{R}_{1}^{4}$. Let $F$ be nondegenerate with normal frame $A$ and let $S$ be endowed with $\Phi_{F}$, then $\sigma_{F}$ is a space-like immersion and $\Omega_{F}$ is the relative induced area form. It follows that if $\sigma_{F}$ is a minimal immersion, then $F$ is $L$-minimal. The converse is also true. In fact, the normal plane to $\sigma_{F}$ at any point $s \in S$ is spanned by $A_{1}(s), A_{4}(s)$. By (1.3), one easily computes that the mean curvatures of $\sigma_{F}$ in the directions $A_{1}$ and $A_{4}$ are, respectively, zero and $p_{1}+p_{3}$ (cf. the conformal analogue in [6]).

2.2 The quartic Abelian differential and a duality theorem for $L$-minimal surfaces. The Laguerre line element $\Phi_{F}$ and the Laguerre area element $\Omega_{F}$ determine on $S$ an oriented conformal structure and hence, by the existence of isothermal coordinates, a unique compatible complex structure characterized by the property that, for any normal frame $A: \mathcal{U} \rightarrow L$, the complex-valued 1-form $\zeta_{A}=\alpha_{0}^{2}+i \alpha_{0}^{3}$ is of type $(1,0)$.

We now define on the Riemann surface $S$ a cross-section $\mathcal{P}(F)$ of the fourth power of the canonical bundle $K(S)$ of $S$ by means of the formula

$$
\mathcal{P}(F)=\left(p_{1}-i p_{2}\right) \zeta_{A}{ }^{4},
$$

and recall the following useful property that can be readily verified (cf. [6]).

(2.13) Let $S$ be a Riemann surface, $\mu$ be a nowhere vanishing 1 -form of type $(1,0)$ defined on an open subset $\mathcal{U} \subset S$. Suppose that $d \mu=-\beta \wedge \mu$ and let $w: \mathcal{U} \rightarrow \mathbb{C}$ be a smooth function. Then $w \mu^{4}$ is a holomorphic section of $K(S)^{4}$ if and only if $d w \equiv 4 w \beta \bmod \mu$.

Theorem 2. Let $F: S \rightarrow \Lambda$ be a nondegenerate Legendre surface. If $F$ is $L$ minimal, then the quartic differential $\mathcal{P}(F)$ is holomorphic.

Proof. Let $A: \mathcal{U} \subset S \rightarrow L$ be a normal frame along $(S, F)$. From (1.30) we obtain

$$
d \zeta_{A}=-\frac{i}{2}\left(q_{1}+i q_{2}\right) \bar{\zeta}_{A} \wedge \zeta_{A}
$$

By substituting $p_{1}=-p_{3}$ into the last two equations of (1.32), we find that

$$
\begin{aligned}
& d p_{1} \wedge \alpha_{0}^{2}+d p_{2} \wedge \alpha_{0}^{3}=-4\left(q_{1} p_{1}+q_{2} p_{2}\right) \alpha_{0}^{2} \wedge \alpha_{0}^{3}, \\
& d p_{2} \wedge \alpha_{0}^{2}-d p_{1} \wedge \alpha_{0}^{3}=-4\left(q_{1} p_{2}-q_{2} p_{1}\right) \alpha_{0}^{2} \wedge \alpha_{0}^{3} .
\end{aligned}
$$

From these equations we derive

$$
d\left(p_{1}-i p_{2}\right) \equiv 2\left[\left(q_{1} p_{2}-q_{2} p_{1}\right)+i\left(q_{1} p_{1}+q_{2} p_{2}\right)\right] \bar{\zeta}_{A} \quad \bmod \zeta_{A} .
$$

Therefore, according to (2.14) and (2.15), it follows that

$$
d\left(p_{1}-i p_{2}\right) \equiv 4\left(p_{1}-i p_{2}\right) \frac{i}{2}\left(q_{1}+i q_{2}\right) \bar{\zeta}_{A} \bmod \zeta_{A},
$$

thereby proving Theorem 2 by virtue of remark (2.13).

We now investigate the properties of the Laguerre transform of an $L$-minimal surface.

A Legendre immersion $F=(f, n): S \rightarrow \Lambda$ is totally degenerate if its spherical map $n$ is constant, i.e., if $f(S)$ lies in a plane of $\mathbb{E}^{3}$. Moreover, an $L$-minimal immersion $F$ is said to be of spherical type if the holomorphic quartic differential $\mathcal{P}(F)$ vanishes identically. Otherwise, we say that $F$ is of general type. For an $L$-minimal surface of general type the zero locus $S_{0}$ of the quartic differential is a discrete subset of $S$. We let $S_{I}$ be its complement. 
Theorem 3. Let $F: S \rightarrow \Lambda$ be a nondegenerate Legendre immersion and let $\check{F}$ : $S \rightarrow \Lambda$ denote its Laguerre transform. Then $F$ is an L-minimal surface of spherical type if and only if $\breve{F}$ is totally degenerate.

Proof. Without loss of generality we may suppose the existence of a global normal frame $A: S \rightarrow L$ along $(S, F)$. Then the Laguerre transform of $F$ is given by

$$
\check{F}: s \in S \mapsto\left[A_{0}(s), A_{4}(s)\right] \in \Lambda .
$$

We next define $\check{A}: S \rightarrow L$ by setting

$$
\check{A}=\left(A_{0}, A_{4}, A_{3}, A_{2}, A_{1}, A_{5}\right) .
$$

This is a Laguerre frame along $\check{F}$ such that, by (1.8), (1.9),

$$
d \check{A}_{1}=-\alpha_{1}^{1} \check{A}_{1}+\alpha_{3}^{1} \check{A}_{2}+\alpha_{2}^{1} \check{A}_{3} .
$$

Observe that $\check{F}$ is totally degenerate if and only if $\left[\check{A}_{1}\right]=$ const. Using $(2.17)$ we derive that $\check{F}$ is totally degenerate if and only if $p_{1}=p_{2}=p_{3}=0$.

As a consequence of this theorem we have

Corollary 4. A nondegenerate Legendrian surface is L-minimal of spherical type if and only if the central spheres are all tangent to a fixed plane in $\mathbb{E}^{3}$.

We will now deal with $L$-minimal surfaces of general type.

Theorem 5. Let $F: S \rightarrow \Lambda$ be an L-minimal surface of general type and let $\check{F}: S \rightarrow \Lambda$ be the Laguerre transform of $F$. Then $\check{F}: S_{I} \rightarrow \Lambda$ is again an $L$ minimal surface of general type and the Laguerre transform of $\check{F}$ coincides with F.

Proof. Pick a normal frame $A: S \rightarrow L$ along $F$ and let $\check{A}: S \rightarrow L$ be the Laguerre frame along $\check{F}$ defined by (2.16). We have

$$
\begin{aligned}
& d \check{A}_{0}=\alpha_{0}^{3} \check{A}_{2}+\alpha_{0}^{2} \check{A}_{3}, \quad d \check{A}_{1}=-\alpha_{1}^{1} \check{A}_{1}+\alpha_{3}^{1} \check{A}_{2}+\alpha_{2}^{1} \check{A}_{3}, \\
& d \check{A}_{2}=-\alpha_{0}^{3} \check{A}_{1}-\alpha_{2}^{3} \check{A}_{2}+\alpha_{3}^{1} \check{A}_{4}+\alpha_{0}^{3} \check{A}_{5}, \\
& d \check{A}_{3}=\alpha_{0}^{2} \check{A}_{1}+\alpha_{2}^{3} \check{A}_{2}+\alpha_{2}^{1} \check{A}_{4}+\alpha_{0}^{2} \check{A}_{5}, \\
& d \check{A}_{4}=-\alpha_{0}^{3} \check{A}_{2}+\alpha_{0}^{2} \check{A}_{3}+\alpha_{1}^{1} \check{A}_{4}, \quad d \check{A}_{5}=0 .
\end{aligned}
$$

Let $\check{\alpha}=\left(\check{\alpha}_{J}^{I}\right)=\check{A}^{-1} d \check{A}$, then by (2.18) we obtain

$$
\left(\begin{array}{c}
\check{\alpha}_{0}^{2} \\
\check{\alpha}_{0}^{3}
\end{array}\right)=\frac{1}{p_{1}^{2}+p_{2}^{2}}\left(\begin{array}{cc}
-p_{1} & p_{2} \\
p_{2} & p_{1}
\end{array}\right)\left(\begin{array}{c}
\check{\alpha}_{1}^{2} \\
\check{\alpha}_{1}^{3}
\end{array}\right)
$$

and

$$
\check{\alpha}_{0}^{1}=0 .
$$

This means that $\check{A}$ is a second order frame along $\check{F}$ and hence the Laguerre transform of $\check{F}$ is

$$
s \in S_{I} \mapsto\left[\check{A}_{0}(s), \check{A}_{4}(s)\right]=\left[A_{0}(s), A_{1}(s)\right]=F(s) .
$$

In order to adapt $\check{A}$ further to a normal frame along $\check{F}$, we consider the functions $d_{2}: S_{I} \rightarrow \mathbb{R}^{+}$and $\theta: S_{I} \rightarrow \mathbb{R}$ such that

$$
p_{1}-i p_{2}=\frac{1}{d_{2}}(\cos 2 \theta-i \sin 2 \theta)
$$


and we define $\check{A}^{\prime}$ by

$$
\check{A}^{\prime}=\check{A} X(d ; b ; 0),
$$

where $d=\left(0, d_{2}\right)$ and $b=\left(\begin{array}{cc}\cos \theta & \sin \theta \\ -\sin \theta & \cos \theta\end{array}\right)$. It is not difficult to verify that $\check{A}^{\prime}$ is a normal frame along $\check{F}$ whose invariant functions satisfy

$$
\check{p}_{1}=-p_{1}, \quad \check{p}_{2}=-p_{2}, \quad \check{p}_{3}=-\check{p}_{1} .
$$

Thus $\check{F}$ is $L$-minimal of general type.

Remark. From the preceding proof we can deduce the following fact: Let $F: S \rightarrow \Lambda$ be a nondegenerate Legendre immersion whose Laguerre transform $\breve{F}$ is nondegenerate. Then $F$ is L-minimal of general type if and only if the Laguerre transform of $\check{F}$ coincides with $F$.

\section{Special examples of Legendre surfaces}

3.1 $\mathrm{L}$-deformable surfaces. The vanishing of $p_{2}$ is connected with the notion of Laguerre deformation for nondegenerate Legendrian surfaces. Two nondegenerate Legendre immersions $F, \tilde{F}: S \rightarrow \Lambda$ of the surface $S$ into $\Lambda$ are Laguerre deformations of each other (or applicable) if $\alpha_{0}^{2}= \pm \tilde{\alpha}_{0}^{2}, \alpha_{0}^{3}= \pm \tilde{\alpha}_{0}^{3}$, where $\left(\alpha_{0}^{2}, \alpha_{0}^{3}\right)$ and $\left(\tilde{\alpha}_{0}^{2}, \tilde{\alpha}_{0}^{3}\right)$ are normal coframings along $F$ and $\tilde{F}$, respectively. We then say that $F$ is deformable if it admits a non-trivial deformation, i.e., a deformation $\tilde{F}$ that is not $L$-equivalent to $F$.

Our definition is related to the general deformation theory of submanifolds in homogeneous spaces ([10], [11]). It may be proved that $F$ and $\tilde{F}$ are deformations of each other if and only if there is a smooth map $B: S \rightarrow L$ with the property that $B(s) \tilde{F}$ and $F$ agree up to order two at $s$, for each $s \in S$. In other words, if and only if $F$ and $\tilde{F}$ are second order deformations with respect to the Laguerre group ([14]).

Proposition 6. Let $F: S \rightarrow \Lambda$ be a nondegenerate Legendre immersion of a simply connected surface. Then $F$ is L-deformable if and only if $p_{2}=0$. Moreover, the non-trivial deformations of $F$ arise in a 1-parameter family.

Proof. Suppose that our surface is $L$-deformable and let $\tilde{F}: S \rightarrow \Lambda$ be a non-trivial deformation of $F$. We may also suppose the existence of global normal Laguerre frames $A$ and $\tilde{A}$ along $F$ and $\tilde{F}$, respectively. Using, if necessary, the $\mathbb{Z}_{2}$-action on normal frames we may also suppose that

$$
\alpha_{0}^{2}=\tilde{\alpha}_{0}^{2}, \quad \alpha_{0}^{3}=\tilde{\alpha}_{0}^{3} .
$$

By the structure equations of normal frames (1.14) and (1.15), (3.1) yields

$$
q_{1}=\tilde{q}_{1}, \quad q_{2}=\tilde{q}_{2}, \quad p_{2}=\tilde{p}_{2}, \quad p_{1}-p_{3}=\tilde{p}_{1}-\tilde{p}_{3} .
$$

Since $\tilde{F}$ and $F$ are not $L$-equivalent, we have

$$
p_{1}+p_{3} \neq \tilde{p}_{1}+\tilde{p}_{3} .
$$

Combining (3.2) and the last two equations of (1.15) we obtain

$$
d\left(p_{1}-\tilde{p}_{1}\right)=2\left(p_{1}-\tilde{p}_{1}\right)\left(q_{1} \alpha_{0}^{3}-q_{2} \alpha_{0}^{2}\right) .
$$


From (3.2) and (3.3) we have $p_{1}-\tilde{p}_{1} \neq 0$ and hence (3.4) implies that $-\alpha_{1}^{1}=$ $2\left(q_{1} \alpha_{0}^{3}-q_{2} \alpha_{0}^{2}\right)$ is closed. On the other hand, the structure equations give $d \alpha_{1}^{1}=$ $2 p_{2} \alpha_{0}^{2} \wedge \alpha_{0}^{3}$. Thus, $p_{2}$ vanishes identically.

Conversely, if $p_{2}=0$ then $\alpha_{1}^{1}$ is closed. Take a smooth function $G$ such that $\alpha_{1}^{1}=-d G$ and define on $S$ the 1-form with values in the Lie algebra of the Laguerre group $L$

$$
\tilde{\alpha}_{C}=\left(\begin{array}{cccccc}
0 & 0 & 0 & 0 & 0 & 0 \\
0 & \alpha_{1}^{1} & \alpha_{2}^{1}+C e^{G} \alpha_{0}^{2} & \alpha_{3}^{1}+C e^{G} \alpha_{0}^{3} & 0 & 0 \\
\alpha_{0}^{2} & \alpha_{0}^{2} & 0 & -\alpha_{2}^{3} & \alpha_{2}^{1}+C e^{G} \alpha_{0}^{2} & 0 \\
\alpha_{0}^{3} & -\alpha_{0}^{3} & \alpha_{2}^{3} & 0 & \alpha_{3}^{1}+C e^{G} \alpha_{0}^{3} & 0 \\
0 & 0 & \alpha_{0}^{2} & -\alpha_{0}^{3} & -\alpha_{1}^{1} & 0 \\
0 & 0 & \alpha_{0}^{2} & \alpha_{0}^{3} & 0 & 0
\end{array}\right)
$$

where $C$ is a constant. The form $\tilde{\alpha}_{C}$ satisfies the compatibility condition

$$
d \tilde{\alpha}_{C}=-\tilde{\alpha}_{C} \wedge \tilde{\alpha}_{C}
$$

By the Cartan-Darboux theorem this equation implies the existence of a mapping $\tilde{A}_{C}: S \rightarrow L$ such that $\tilde{A}_{C}^{-1} d \tilde{A}_{C}=\tilde{\alpha}_{C}$. Moreover, $\tilde{A}_{C}$ is unique up to multiplication by an element of $L$. Thus, if we set

$$
\tilde{F}_{C}: s \in S \rightarrow\left[\tilde{A}_{C, 0}(s), \tilde{A}_{C, 1}(s)\right],
$$

then $\tilde{F}_{C}$ is a non-trivial deformation of $F$ and

$$
\tilde{p}_{C, 1}=p_{1}+C e^{G}, \quad C \in \mathbb{R}, C \neq 0 .
$$

From the first part of the proof (cf. (3.4)) it follows that, if $\tilde{F}$ is any non-trivial Laguerre deformation of $F$, then

$$
\tilde{p}_{1}=p_{1}+C e^{G}
$$

for some constant $C \neq 0$. Thus, $\tilde{A}^{-1} d \tilde{A}=\tilde{\alpha}_{C}$ and hence $\tilde{F}$ is congruent to the deformation $\tilde{F}_{C}$. This shows that the non-trivial deformations of $F$ are parameterized, up to $L$-equivalence, by non-zero real numbers.

3.2 $L$-minimal deformable surfaces. The invariant functions $q_{i}$ and $p_{j}$ of an $L$-minimal deformable surface satisfy

$$
p_{1}+p_{3}=0, \quad p_{2}=0 .
$$

We observe that the sign of $p_{1}$ is independent of the choice of normal frame. Accordingly, we have three cases to consider $p_{1}<0, p_{1}=0, p_{1}>0$, respectively.

Proposition 7. Let $F: S \rightarrow \Lambda$ be a L-minimal immersion of a simply connected surface into $\Lambda$ and assume $p_{1}<0$. Then $F$ is L-deformable if and only if $F$ is $L$-equivalent to the Legendrian lift of a minimal immersion of $S$ in $\mathbb{E}^{3}$ without parabolic points.

Proof. Let $A: S \rightarrow \Lambda$ be a normal frame along $F$. According to (1.15),

$$
\alpha_{1}^{1}=-\frac{d p_{1}}{2 p_{1}} .
$$

We further adapt $A$ to another Laguerre frame

$$
B=A X\left(d ; I_{2}, 0\right),
$$


where $d=\left(0, \sqrt{-p_{1}}\right)$. By setting $\beta=B^{*}(\omega)$, we find

$$
\beta=\left(\begin{array}{cccccc}
0 & 0 & 0 & 0 & 0 & 0 \\
0 & 0 & -\sqrt{-p_{1}} \alpha_{0}^{2} & \sqrt{-p_{1}} \alpha_{0}^{3} & 0 & 0 \\
\alpha_{0}^{2} & \sqrt{-p_{1}} \alpha_{0}^{2} & 0 & -\alpha_{2}^{3} & -\sqrt{-p_{1}} \alpha_{0}^{2} & 0 \\
\alpha_{0}^{3} & -\sqrt{-p_{1}} \alpha_{0}^{3} & \alpha_{2}^{3} & 0 & \sqrt{-p_{1}} \alpha_{0}^{3} & 0 \\
0 & 0 & \sqrt{-p_{1}} \alpha_{0}^{2} & -\sqrt{-p_{1}} \alpha_{0}^{3} & 0 & 0 \\
0 & 0 & \alpha_{0}^{2} & \alpha_{0}^{3} & 0 & 0
\end{array}\right) .
$$

This shows that $\beta$ takes values in the Euclidean subalgebra of the Lie algebra $\ell$ of $L$. This means that, up to left translations by elements of $L$, the map $B$ sends $S$ into the Euclidean subgroup $\mathbb{E}(3)$ in $L$. Therefore, by (3.7) and (1.16), $F$ is the Legendrian lift of a surface $f: S \rightarrow \mathbb{E}^{3}$, whose principal curvatures $\kappa_{2}$ and $\kappa_{3}$ are given by

$$
\kappa_{2}=-\sqrt{-2 p_{1}}, \quad \kappa_{3}=\sqrt{-2 p_{1}} .
$$

From this we deduce that $f$ is a minimal surface. Conversely, if $F: S \rightarrow \Lambda$ is the Legendrian lift of a minimal surface $f: S \rightarrow \mathbb{E}^{3}$, we may use a principal orthonormal framing along $f$ to define a Laguerre frame $B: S \rightarrow L$ along $F$ such that $B^{-1} d B$ has the form (3.7). We thus set $A=B X\left(d ; I_{2} ; 0\right)^{-1}$ to get a normal frame along $F$ whose invariant functions satisfy (3.6).

Proposition 8. Let $F: S \rightarrow \Lambda$ be a L-minimal immersion of spherical type $\left(p_{1}=\right.$ $\left.p_{2}=p_{3}=0\right)$ of a simply connected surface $S$ into $\Lambda$. Then, up to L-equivalence, $F$ is given by

$$
F(s)=\left[F_{0}(s), F_{1}(s)\right], \text { for each } s \in S,
$$

where $F_{0}$ and $F_{1}$ are $\mathbb{R}^{6}$-valued mappings of the form

$$
\begin{aligned}
& F_{0}={ }^{t}\left(1,0, \mu_{1}, \mu_{2}, \int\left(\rho_{1} d \mu_{1}-\rho_{2} d \mu_{2}\right), \frac{1}{2}\left(\mu_{1}{ }^{2}+\mu_{2}{ }^{2}\right)\right) \\
& F_{1}={ }^{t}\left(0,1, \rho_{1},-\rho_{2}, \frac{1}{2}\left(\rho_{1}{ }^{2}+\rho_{2}{ }^{2}\right), \int\left(\mu_{1} d \rho_{1}-\mu_{2} d \rho_{2}\right)\right),
\end{aligned}
$$

where $\mu=\mu_{1}+i \mu_{2}$ and $\rho=\rho_{1}+i \rho_{2}$ are holomorphic functions, $d \mu \wedge d \bar{\rho}_{\left.\right|_{s}} \neq 0$, for each $s \in S$, and

$$
\sigma_{1}=\int\left(\rho_{1} d \mu_{1}-\rho_{2} d \mu_{2}\right), \quad \sigma_{2}=\int\left(\mu_{1} d \rho_{1}-\mu_{2} d \rho_{2}\right)
$$

are the primitives of the closed 1 -forms $\rho_{1} d \mu_{1}-\rho_{2} d \mu_{2}$ and $\mu_{1} d \rho_{1}-\mu_{2} d \rho_{2}$ satisfying

$$
\rho_{1} \mu_{1}-\rho_{2} \mu_{2}-\sigma_{1}-\sigma_{2}=0 .
$$

Proof. Let $A: S \rightarrow L$ be a normal frame along $F$. Since $p_{1}=p_{2}=p_{3}=0$, we have $\alpha_{2}^{1}=\alpha_{3}^{1}=0$ and $\alpha_{1}^{1}, \alpha_{2}^{3}$ are closed 1-forms. Fix functions $y, w: S \rightarrow \mathbb{R}$ such that

$$
\alpha_{1}^{1}+\frac{d y}{y}=\alpha_{2}^{3}+d w=0
$$


Let $X: S \rightarrow L_{0}$ be defined by $X=X(d ; b ; 0)$, where $d=(0, y)$ and $b=e^{-i w}$. Consider the Laguerre frame $B=A X$ and set $\beta=B^{-1} d B$. We then have

$$
\beta=\left(\begin{array}{cccccc}
0 & 0 & 0 & 0 & 0 & 0 \\
0 & 0 & 0 & 0 & 0 & 0 \\
\beta_{0}^{2} & \beta_{1}^{2} & 0 & 0 & 0 & 0 \\
\beta_{0}^{3} & \beta_{1}^{3} & 0 & 0 & 0 & 0 \\
0 & 0 & \beta_{1}^{2} & \beta_{1}^{3} & 0 & 0 \\
0 & 0 & \beta_{0}^{2} & \beta_{0}^{3} & 0 & 0
\end{array}\right),
$$

where $\beta_{0}^{2}+i \beta_{0}^{3}$ and $\beta_{1}^{2}-i \beta_{1}^{3}$ are holomorphic 1-forms on $S$. Let $\mu=\mu_{1}+i \mu_{2}$ and $\rho=\rho_{1}+i \rho_{2}$ be holomorphic functions such that

$$
d \mu=\beta_{0}^{2}+i \beta_{0}^{3}, \quad d \rho=\beta_{1}^{2}-i \beta_{1}^{3} .
$$

The column vectors $B_{J}$ of the frame $B$ satisfy the equations

$$
\begin{aligned}
& d B_{0}=d \mu_{1} B_{2}+d \mu_{2} B_{3}, \\
& d B_{1}=d \rho_{1} B_{2}-d \rho_{2} B_{3}, \\
& d B_{2}=d \rho_{1} B_{4}+d \mu_{1} B_{5}, \\
& d B_{3}=-d \rho_{2} B_{4}+d \mu_{2} B_{5}, \\
& d B_{4}=0, \quad B_{5}=e_{5} .
\end{aligned}
$$

Thus, $B_{4}$ is constant and

$$
\left\|B_{4}\right\|^{2}=\left\langle B_{4}, e_{5}\right\rangle=0
$$

From the third and fourth equation of (3.9) we obtain

$$
B_{2}=C_{2}+\rho_{1} B_{4}+\mu_{1} B_{5}, \quad B_{3}=C_{3}-\rho_{2} B_{4}+\mu_{2} B_{5},
$$

where $C_{2}$ and $C_{3}$ are constant vectors satisfying

$$
\begin{aligned}
& \left\|C_{2}\right\|^{2}=\left\|C_{3}\right\|^{2}=1, \\
& \left\langle C_{2}, C_{3}\right\rangle=\left\langle C_{2}, B_{4}\right\rangle=\left\langle C_{2}, B_{5}\right\rangle=0, \\
& \left\langle C_{3}, B_{4}\right\rangle=\left\langle C_{3}, B_{5}\right\rangle=0 .
\end{aligned}
$$

Combining (3.10) and the first two equations in (3.9) we obtain

$$
\begin{aligned}
& B_{0}=C_{0}+\mu_{1} C_{2}+\mu_{2} C_{3}+\sigma_{1} B_{4}+\frac{1}{2}\left(\mu_{1}^{2}+\mu_{2}^{2}\right) B_{5}, \\
& B_{1}=C_{1}+\rho_{1} C_{2}-\rho_{2} C_{3}+\frac{1}{2}\left(\rho_{1}^{2}+\rho_{2}^{2}\right) B_{4}+\sigma_{2} B_{5},
\end{aligned}
$$

where $C_{0}$ and $C_{1}$ are constant vectors such that $C=\left(C_{0}, C_{1}, C_{2}, C_{3}, B_{4}, B_{5}\right)$ is an element of the Laguerre group. Setting $F^{\prime}=C^{-1} F$ we then have that $F^{\prime}$ is given by formula (3.8).

For the case $p_{1}>0$ we make the following remark without entering too much into details.

Remark. a) If $p_{1}>0$ and $p_{2}=0$, then the central congruence of spheres is a maximal space-like immersion into a 3-dimensional subspace of $\mathcal{Q}_{\Sigma}$ of signature $(2,1)$ (cf. [12]).

b) Another class of $L$-minimal deformable immersions is given by the $L$-minimal canal surfaces. They are studied in [13], where the integration problem is solved and explicit expressions for the solution surfaces are provided. 


\section{REFERENCES}

1. L. Bianchi, Opere, v. XI, Corrispondenza, pp. 199-202, Cremonese, Roma, 1959. MR 24:A27

2. W. Blaschke, Über die Geometrie von Laguerre: I. Grundformeln der Flächentheorie, Abh. Math. Sem. Univ. Hamburg 3 (1924), 176-194.

3. __ Über die Geometrie von Laguerre: II. Flächentheorie in Ebenenkoordinaten, Abh. Math. Sem. Univ. Hamburg 3 (1924), 195-212.

4. _ـ Über die Geometrie von Laguerre: III. Beiträge zur Flächentheorie, Abh. Math. Sem. Univ. Hamburg 4 (1925), 1-12.

5. __ Vorlesungen über Differentialgeometrie und geometrische Grundlagen von Einsteins Relativitätstheorie, B. 3, bearbeitet von G. Thomsen, J. Springer, Berlin, 1929.

6. R.L. Bryant, A duality theorem for Willmore surfaces, J. Differential Geom. 20 (1984), 23-53. MR 86j: 58029

7. R.L. Bryant, S.S. Chern, R.B. Gardner, H.L. Goldschmidt, P.A. Griffiths, Exterior differential systems, Mathematical Science Research Institute Publications, Springer-Verlag, New York, Berlin, Heidelberg, 1991. MR 92h:58007

8. E. Cartan, Théorie des groupes finis et continus et la géométrie différentielle traitées par la méthode du repère mobile, Gauthier-Villars, Paris, 1937.

9. T.E. Cecil, Lie sphere geometry: with applications to submanifolds, Springer-Verlag, New York, 1992. MR 94m:53076

10. P.A. Griffiths, On Cartan's method of Lie groups and moving frames as applied to uniqueness and existence questions in differential geometry, Duke Math. J. 41 (1974), 775-814. MR 53:14355

11. G.R. Jensen, Deformation of submanifolds in homogeneous spaces, J. Differential Geom. 16 (1981), 213-246. MR 83h:53063

12. T. Klotz-Milnor, Harmonic maps and classical surface theory in Minkowski 3-space, Trans. Amer. Math. Soc. 280 (1983), 161-185. MR 85e:58037

13. E. Musso, L. Nicolodi, L-minimal canal surfaces, Rend. Matematica 15 (1995), 421-445. CMP 96:04

14. E. Musso, L. Nicolodi, Isothermal surfaces in Laguerre geometry, Boll. Un. Mat. Ital. (to appear).

Dipartimento di Matematica Pura ed Applicata, Università di L'Aquila, via Vetoio, I-67010 Coppito, L' Aquila, Italy

E-mail address: musso@axscaq.aquila.infn.it

Dipartimento di Matematica "G. Castelnuovo", Università di Roma "La Sapienza", P.LE A. Moro 2, I-00185 Roma, Italy

E-mail address: nicolodi@mat.uniroma1.it 